Originalien

Unfallchirurg 2022 $125: 219-226$

https://doi.org/10.1007/s00113-021-01009-3

Angenommen: 14. April 2021

Online publiziert: 8. Juni 2021

(c) Der/die Autor(en) 2021

\section{Redaktion}

W. Mutschler, München

H. Polzer, München

B. Ockert, München

\section{Einführung}

Die Gestaltung der medizinischen Lehre ist seit jeher eine Herausforderung für Studierende und Lehrende. Ein Großteil der medizinischen Lehre erfolgt hierbei auch heute noch über eher dozentenzentrierte Lehrmodelle, z. B. durch Vorlesungen [13]. Allerdings steigt die Bedeutung in der medizinischen Lehre für studierendenzentrierte Lehrmethoden gegenüber den traditionellen dozentenzentrierten Modellen, da hierdurch der Lehrinhalt praxisnäher gestaltet werden kann [7]. Durch die Förderung des Zusammenhangswissens soll der Wissenstransfer von der Theorie in die Praxis besser gelingen [7, 21].

Eine Möglichkeit hierfür ist der Einsatz von fallbasiertem Lernen. Hierbei bearbeiten Studierende strukturierte Fallbeispiele. Der Lehrende nimmt in der anschließenden Diskussion die Rolle des Moderators ein, um diese entsprechend zu steuern [7]. Dadurch kann der Lehrende Einfluss auf den Fokus relevanter Aspekte nehmen und gewährleistet

Aus Gründen der leichteren Lesbarkeit wird in dem vorliegenden Artikel die männliche Sprachform verwendet. Jedoch sind hiermit alle Geschlechter einbezogen.

Friedemann Strobel ${ }^{1,2}$ - Tina Histing ${ }^{1}$ Tim Pohlemann ${ }^{1}$ Antonius Pizanis ${ }^{1} \cdot$ Benedikt Johannes Braun ${ }^{1} \cdot$ Marcel Orth ${ }^{1} \cdot$ Tobias Fritz $^{1}$

${ }^{1}$ Klinik für Unfall-, Hand- und Wiederherstellungschirurgie, Universitätsklinikum des Saarlandes, Homburg/Saar, Deutschland

${ }^{2}$ Klinik für Anästhesiologie und operative Intensivmedizin, Kliniken Köln-Merheim, Köln, Deutschland

\title{
Fallbasiertes Lernen kann die Lehrqualität im unfallchirurgischen Unterricht verbessern
}

\section{Eine Umfrageanalyse unter Medizinstudierenden}

einen strukturierten und effizienten $\mathrm{Ab}$ lauf der Falllösung [7].

Um die positiven Effekte des fallbasierten Lernens zu nutzen und dadurch eine weniger vom Dozenten abhängige Lehre zu erreichen, war es das Ziel dieser Arbeit, diese in das unfallchirurgische Curriculum zu integrieren.

Im Rahmen des unfallchirurgischen Praxisseminars des Studiengangs $\mathrm{Hu}-$ manmedizin soll praxisnahes Wissen vermittelt werden. Bis zur Umstellung auf das hier beschriebene fallbasierte Lernen war das unfallchirurgische Praxisseminar in 6 Unterrichtsthemen mit je $90 \mathrm{~min} 2$-mal/Woche im 1 . bzw. 2. klinischen Semester etabliert. Abschließend erfolgte eine Gesamtklausur über die Inhalte aller chirurgischen Praxisseminare (Allgemein-, Viszeral-, Gefäß-, Kinder-, Neuro-, Herz-, Thoraxund Unfallchirurgie). Das Praxisseminar Unfallchirurgie konnte daher nicht eigenständig auf andere Prüfungsmodalitäten umgestellt werden (z. B. OSCE). Der Umstellung auf fallbasiertes Lernen war eine Analyse der eingesetzten Unterrichtsmaterialien der Dozenten des Praxisseminars vorangegangen. Hierbei zeigten sich strukturelle und inhaltliche Unterschiede. Bis zur Restrukturierung war es den Dozenten freigestellt, welchen Lehransatz und welche Lehrmittel
Infobox 1 Zusammenstellung der Kasuistikthemen

Obere Extremität und Thorax

Schulterluxation

Proximale Humerusfraktur - einfacher

Frakturtyp

Proximale Humerusfraktur - mehrfragmentärer Frakturtyp

Pneumothorax

Untere Extremität

Tibiakopffraktur

Mediale Schenkelhalsfraktur

Pertrochantäre Femurfraktur

Unterschenkelfraktur

Hand und Handgelenk

Distale Radiusfraktur

Distale Radiusfraktur des Kindes

Mittelhandfraktur

Skaphoidfraktur

Wirbelsäule und Becken

Densfrakturen

Fraktur der LWS/BWS

Beckenfraktur

Beckenfraktur mit kombinierter Acetabulum-

fraktur

Fuß und Sprunggelenk

Sprunggelenkluxationsfraktur

Kalkaneusfraktur

Lisfranc-Luxation

Talusfraktur 


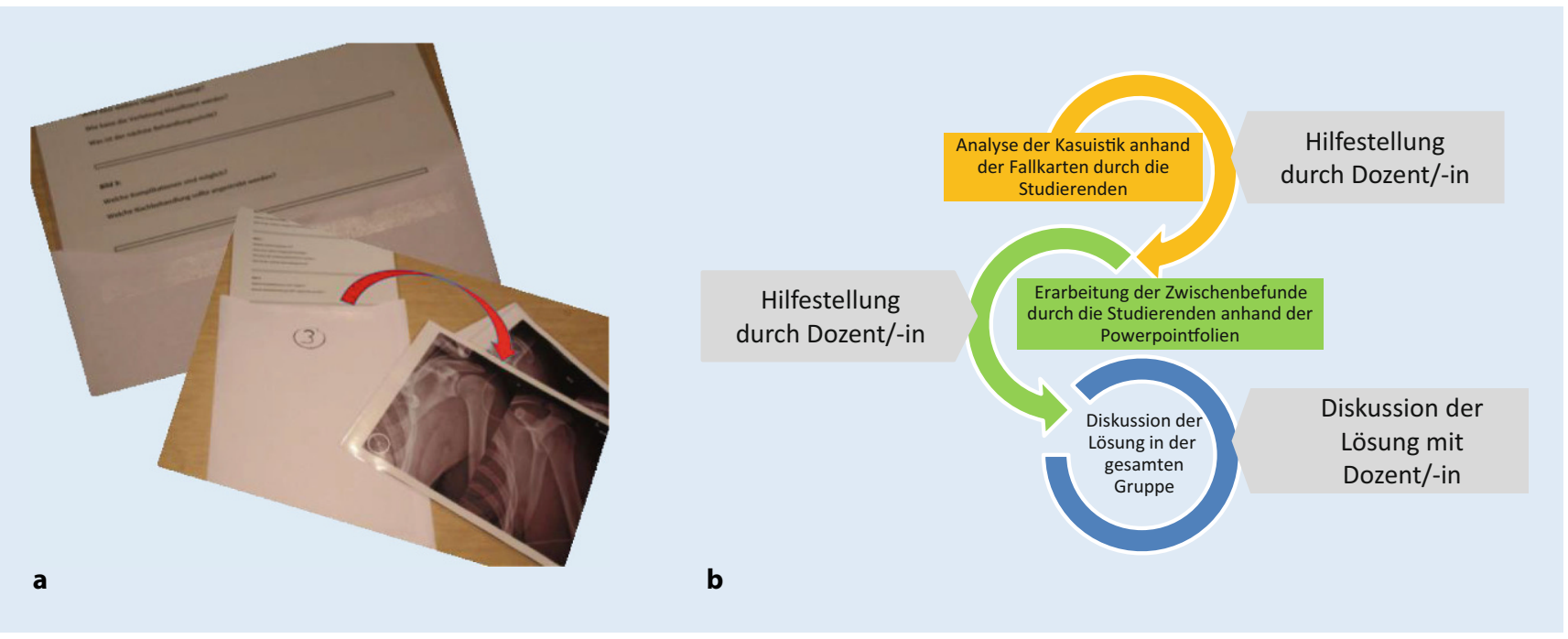

Abb. $1 \Delta$ Exemplarische Darstellung der papierbasierten Kasuistiken (a) sowie des Seminarablaufs (b)

sie verwenden wollten. Lediglich das vorgegebene Themengebiet sollte behandelt werden. Eine standardisierte Ausbildung der Studierenden konnte damit nicht in allen Fällen gewährleistet werden. Fallbasiertes Lernen und problemorientiertes Lernen (POL) wurden am Lehrstuhl als freiwilliges Lehrangebot angeboten. Die darin enthaltenen Inhalte wurden außerdem für eine internetbasierte Lernplattform im Sinne des distributiven problemorientierten Lernens (dPOL) verwendet [3, 11, 12].

Ziel des Projektes war es, für alle Seminarteilnehmer eine äquivalente, qualitativ hochwertige Lehre zu gewährleisten. Darüber hinaus sollten Dozenten mit unterschiedlicher Berufserfahrung eine Struktur zur Verfügung gestellt werden, die eine solche Lehre sicherstellt. Ein Ansatz, um dies zu erreichen, ist die Etablierung eines dozenten-/ studierendenzentrierten balancierten Lehrkonzepts mit fallbasiertem Lernen.

\section{Material und Methoden}

\section{Kasuistiken}

Anhand der DGUV-Unfallstatistik 2016 [26] wurden häufige und für den klinischen Alltag repräsentative Verletzungsarten ausgewählt. Diese wurden anschließend als repräsentative anonymisierte Fallbeispiele ausgearbeitet (•Infobox 1). $\mathrm{Zu}$ den einzelnen Fallbeispielen wurden
Bearbeitungsanleitungen und Klassifikationshilfen erstellt. Gegliedert war jedes Fallbeispiel in die Abschnitte Anamnese/ Fallbeschreibung und Diagnostik (inklusive anonymisierter Röntgen-, CTund MRT-Bilder). Aus den Unterlagen wurden entsprechende Fallkarten im DIN-A4-Format ausgedruckt und den Studierenden für das Seminar zur Verfügung gestellt (• Abb. 1). Außerdem wurde für jedes Fallbeispiel eine Kasuistik als Präsentation (PowerPoint; Fa. Microsoft, Redmond, WA, USA) erstellt.

\section{Seminarablauf}

Der Unterricht umfasste 7 Termine mit je 90 min während des Wintersemesters 2017/2018. Hierbei waren 2 Termine für praktische Inhalte wie Nahtkurs und Untersuchungstechniken vorgesehen. Die weiteren 5 Termine waren als Seminar geplant. Die Dozenten $(n=20)$ waren klinisch tätige Ärztinnen und Ärzte der Unfallchirurgie vom 1. bis 28. Berufsjahr. Vor Durchführung des Semesters erfolgte eine 60-minütige Instruktion aller Dozenten über die neue Seminarstruktur. Das Praxisseminar war in Gruppen mit je $20 \pm 2$ Studierenden aufgeteilt. Innerhalb der Seminargruppe wurden, entsprechend der Literaturempfehlung, Kleingruppen zu je 4 bis 5 Personen gebildet $[16,23]$. Jeder Gruppe wurde ein Fall zugewiesen. Innerhalb von $15 \mathrm{~min}$ sollten eine Verdachtsdiagnose gestellt und Vorschläge für eine fachgerechte Diagnostik, Klassifikation und Therapie entwickelt werden. Fragen an bzw. Hilfestellung durch die Dozenten waren jederzeit möglich. Nach erfolgreicher Bearbeitung der Fälle erfolgte durch die Studierenden eine Vorstellung der Kasuistik mithilfe der Fallpräsentationen.

\section{Evaluation/Auswertung}

Ein standardisierter Evaluationsbogen der Universität für Seminare (• Infobox 2) wurde bereits im Sommersemester 2016 verwendet, in welchem der Unterricht nach alten Vorgaben ohne einheitliche Kasuistik erfolgte und diente als Kontrollgruppe. Der gleiche Evaluationsbogen des Sommersemesters 2016 wurde mit dem des Wintersemesters 2017/2018 verglichen (•Infobox 2). Darüber hinaus wurde für das Projekt ein zusätzlicher Evaluationsbogen erstellt und durch die Studierenden nach Abschluss des Semesters beantwortet ( Infobox 3). Das Ziel war es, zunächst Kollektivdaten wie Alter und Geschlecht zu evaluieren (- Infobox 3). Dann wurden die Berufswunsch-Präferenzen der Studierenden erfragt (•Infobox 3). Abschließend erfolgten spezifische Fragen bezüglich des Seminarkonzeptes (- Infobox 3).

Die Fragen wurden so konzipiert, dass sie mit einer klaren Ja- oder Nein-Antwort beantwortet werden konnten, um 
eine Ablehnung oder Befürwortung klar zu definieren.

\section{Statistik}

Die statistische Auswertung erfolgte mittels Microsoft Excel(Fa. Microsoft, Redmond, WA, USA) und Sigma Plot 13 (Fa. Systat, San José, CA, USA). Die Analyse zwischen den Gruppen erfolgte mittels zweiseitigem $t$-Test; dieser wurde bei signifikantem Unterschied um einen einseitigen $t$-Test ergänzt. Das Signifikanzniveau wurde mit $p<0,05$ festgelegt.

\section{Ergebnisse}

Insgesamt zeigte sich die Anzahl ausgewerteter Evaluationsbogen der Universität mit $n_{2017}=80$ vs. $n_{2016}=116$. Die standardisierten Fragen waren in beiden Jahrgängen gleich erstellt, lediglich die Reihenfolge auf dem Evaluationsbogen wich voneinander ab. Das Curriculum war in beiden Jahrgängen vor dem ersten Termin des Praxisseminars offiziell bereitgestellt, sodass die Studierenden dieses auch in beiden Jahren bestätigten. In der Auswertung der beiden unterschiedlichen Semester zeigten sich signifikant bessere Bewertungen des neu strukturierten Seminars für die Veranstaltung insgesamt, der Definition der Lernziele, der Prüfungsrelevanz, der Motivation und Vorbereitung des Dozenten, der Wiederholung der Inhalte, der Bereitschaft zur Diskussion und des Tempos des Dozenten (• Tab. 1). In der Gruppe der Studierenden des neu strukturierten Seminars wurde die fachliche Kompetenz der Dozenten signifikant höher eingeschätzt $\left(\mathrm{M}_{2016}=2,03, \mathrm{SD}=0,9\right.$ vs. $\left.\quad \mathrm{M}_{2017}=1,6, \quad \mathrm{SD}=0,67 ; \quad p<0,001\right)$ (- Abb. 2a). Auch die Lernatmosphäre wurde durch das neue Kurskonzept verbessert $\left(\mathrm{M}_{2016}=2,31 ; \mathrm{SD}=0,98\right.$ vs. $\quad \mathrm{M}_{2017}=1,94 ; \quad \mathrm{SD}=1,0 ; \quad p=0,006$ ) (• Abb. 2b). Keine signifikanten Unterschiede zeigten sich für den Bezug zu aktuellen Themen sowie die Qualität der Unterrichtsmaterialien und der fächerübergreifenden Lehre (•Tab. 1).

An der selbsterstellten Evaluation nahmen insgesamt 130 Studierende teil, die sich durchschnittlich im 6. Studiensemester befanden und das neu gestal-

Unfallchirurg 2022 $\cdot 125: 219-226$ https://doi.org/10.1007/s00113-021-01009-3

(c) Der/die Autor(en) 2021

F. Strobel · T. Histing · T. Pohlemann · A. Pizanis · B. J. Braun · M. Orth · T. Fritz

\section{Fallbasiertes Lernen kann die Lehrqualität im unfallchirurgischen Unterricht verbessern. Eine Umfrageanalyse unter Medizinstudierenden}

\section{Zusammenfassung}

Hintergrund. Medizinische Lehre ist seit jeher eine Herausforderung für Studierende und Dozenten. Die Förderung des Zusammenhangswissens und ein Wissenstransfer von der Theorie auf die Praxis gewinnen in den letzten Jahren an Bedeutung. Um dieses Ziel zu erreichen, werden zunehmend studierendenzentrierte Lehrkonzepte in der Literatur eingesetzt.

Fragestellung. Kann durch ein fallbasiertes Lehrkonzept das unfallchirurgische Seminar verbessert werden?

Material und Methoden. Den Studierenden und Dozenten wurden standardisierte Fallbeispiele und dazugehörige Unterrichtsmaterialien wie Klassifikationshilfen und Versorgungsstrategien zur Verfügung gestellt. Durch eine zweizeitige Evaluation konnten die Auswirkungen dieser Modifikationen des Lehrdesigns erfasst und statistisch ausgewertet werden.

Ergebnisse. Das Seminar wurde als prüfungsrelevanter empfunden. Die Lehre durch die Dozenten wurde als kompetenter und motivierter empfunden. Insgesamt wurde das Seminar durch die Studierenden besser bewertet.

Schlussfolgerungen. Ein fallbasiertes Lehrkonzept kann daher, richtig und gezielt eingesetzt, die unfallchirurgische Lehre signifikant verbessern.

\section{Schlüsselwörter}

Chirurgische Lehre · Fallbasiertes Lernen . Studierendenzentriertes Lehrformat · Seminar Unfallchirurgie - Lehre

\section{Case-based learning can improve the teaching quality in trauma surgery education. A survey analysis among medical students}

\section{Abstract}

Background. Medical education has always been challenging for students and teachers. Of growing importance is the promotion of knowledge of correlations and knowledge transfer from theoretical aspects into clinical practice. In order to achieve this aim, studentcentered teaching concepts are increasingly being employed in the literature.

Objective. Can a trauma surgery practical seminar be improved by a case-based teaching concept?

Material and methods. For this purpose, standardized case studies and corresponding teaching materials, such as classification aids and treatment strategies, were made available to the students and lecturers. Using a two-staged evaluation the effects of the modified teaching design could be recorded and statistically analyzed.

Results. The seminar was considered to be relevant for the examinations. The teaching by the lecturers was found to be more competent and appeared more motivated. Overall, the seminar was rated better by the students.

Conclusion. A case-based teaching concept can significantly improve the education in trauma surgery, when correctly and specifically implemented.

Keywords

Surgical teaching - Case-based learning . Student-centered teaching format - Trauma surgery seminar . Teaching tete Seminar absolviert hatten (41\% männlich zu 59\% weiblich). Der Berufswunsch der Studierenden nach Abschluss des Seminars zeigte, dass $24 \%$ ein nichtoperatives Fach wählen wollten, $40 \%$ es noch nicht wussten und $36 \%$ eine Weiterbildung in einem operativen Fach machen wollten (- Abb. 3a). Insgesamt fanden $76 \%$ der Studierenden den Lerninhalt durch verschiedene
Dozenten reproduzierbar (• Abb. $3 \mathbf{b})$. Dem Großteil der Studierenden gefiel das fallbasierte Lernen in der Gruppe (- Abb. 3c). Das Praxisseminar wurde in $54 \%$ der Fälle mithilfe der erstellten Fallkarten durchgeführt; in $92 \%$ der Fälle wurden die erstellten Fallpräsentationen genutzt (• Abb. 3d). Die Frage „Fanden Sie das Praxisseminar Unfallchirurgie gut strukturiert?" wurde in 


\section{Infobox 2 Evaluationsbogen der Universität zum semesterübergrei- fenden Vergleich}

\section{Evaluationsbogen 1}

Wie bewerten Sie die Veranstaltung insgesamt?

Inwieweit wurden Bezüge zu aktuellen

Themen hergestellt?

Inwieweit wurden medizinische/

zahnmedizinische/klinische Bezüge

hergestellt?

Wie gut wurden die angegebenen Lernziele definiert?

Inwieweit schätzen Sie die Veranstaltung prüfungsrelevant ein?

Wie bewerten Sie die Qualität der

Unterrichtsmaterialien und -medien?

Wie schätzen Sie die fachliche Kompetenz

des/der Dozenten ein?

Wie bewerten Sie die Motivation und

Vorbereitung des/der Dozenten?

Konnten Sie dem Tempo des Dozenten gut folgen?

Hat der Dozent die Inhalte angemessen

wiederholt?

Wie bewerten Sie die Möglichkeit, Fragen zu stellen, und die Bereitschaft zur Diskussion? Wie gut wurde fächerübergreifend gelehrt? Wie empfanden Sie die entstandene Lernund Arbeitsatmosphäre?

$70 \%$ mit ja beantwortet (30\% nein). Die Anforderungen des Praxisseminars wurden von $79 \%$ der Studierenden als „genau richtig“ empfunden. $17 \%$ waren unterfordert und $4 \%$ überfordert. Die Themen bezüglich der klinischen Relevanz wurden überwiegend mit sehr gut (14\%) und gut (58\%) bewertet. $20 \%$ empfanden die Relevanz befriedigend, $7 \%$ ausreichend und 1\% mangelhaft. Durch das Praxisseminar konnten 54\% der Studierenden für das Fach Unfallchirurgie/Orthopädie motiviert werden. $23 \%$ der Studierenden hatten vor dem Praxisseminar die Hauptvorlesung „Unfallchirurgie“ besucht. Weiter wurde auch im spezifischen zweiten Evaluationsbogen die neue Struktur in Schulnoten bewertet; hier ergab sich die Note $2,46(\mathrm{SD}=0,79)$. Hierbei zeigte sich in der Subgruppenanalyse (Hauptvorlesung besucht vs. nicht besucht) kein Unterschied in der Benotung zwischen den Studierenden, die die Hauptvorlesung besucht hatten $(2,43 \mathrm{SD}=0,93$; $p>0,05)$, und der Bewertung der Gruppe der Studierenden, welche die Vorlesung nicht besucht hatten $(2,5 \mathrm{SD}=0,735$;

\section{Infobox 3 Für das Projekt entwickelter, zusätzlicher Evaluationsbogen}

\section{Evaluationsbogen 2}

Welche Erwartungen hatten Sie an das

Praxisseminar Unfallchirurgie?

Was ist Ihr Berufsziel?

Welche Erwartungen hätten Sie an den Kurs, wenn Sie ihn veranstalten würden?

Semester

Geschlecht

Alter

Wie schätzen Sie Ihre handwerkliche Geschicklichkeit ein?

Wie fanden Sie die gewählten Themen für den Kurs bezüglich ihrer klinischen Relevanz? Wie empfanden Sie die Anforderungen des Praxisseminars?

Hat Ihnen das fallbasierte Lernen in der Gruppe gefallen?

Fanden Sie das Praxisseminar Unfallchirurgie gut strukturiert?

Fanden Sie das Praxisseminar Unfallchirurgie unabhängig vom durchführenden Dozenten standardisiert?

Fanden bzw. können Sie sich vorstellen, dass die vermittelten Lehrinhalte durch unterschiedliche Dozenten reproduzierbar sind?

Wurde das Praxisseminar mit den erstellten Lernkarten (Din-A4-Umschlägen mit Gruppenarbeit) durchgeführt?

Der Unterricht wurde anhand der

standardisierten PowerPoint-Fälle

durchgeführt?

- Fanden Sie die entstandene Lernatmosphäre gut?

- Hätten Sie sich mehr aktive Mitarbeit gewünscht?

- Wenn Ja, wie?

- Konnten Sie den Lerninhalten folgen?

Hat Sie der Kurs animiert, sich für das Fach Orthopädie/Unfallchirurgie zu begeistern? Hatten Sie vor dem Kurs bereits die Vorlesung "Unfallchirurgie" besucht?

Halten Sie es für sinnvoller, das Praxisseminar in einem höheren Semester (nach dem

Abschluss der Hauptvorlesung Chirurgie) ins Curriculum einzubinden?

Welche Schulnote würden Sie dem neu strukturierten Praxisseminar Unfallchirurgie geben?

Haben Sie Verbesserungsvorschläge? Wenn ja, welche:

$p>0,05)(\bullet$ Abb. 3e). Die Subgruppenanalyse zeigte außerdem: Studierende, welchen das fallbasierte Konzept gefiel, zeigten keinen Unterschied zwischen dem Einsatz von Fallkarten und ohne Fallkarten. Es zeigte sich auch kein Unterschied zwischen Studierenden, welche die Hauptvorlesung besucht hatten, und den Studierenden, welche die Hauptvorlesung nicht besucht hatten (• Abb. 3f).

\section{Diskussion}

Durch ein fallbasiertes Lehrkonzept für das unfallchirurgische Praxisseminar konnte dieses zu einer eher studierendenzentrierten Lehrveranstaltung verändert werden. Die schrittweise Analyse eines klinischen Falles und die Verknüpfung mit bereits vorhandenem Wissen sind dem klinischen Arbeitsalltag ähnlicher als klassische Lernmethoden wie z. B. Frontalunterricht. So wurden bereits verschiedene studierendenzentrierte Curricula entwickelt und publiziert $[1,2,4,10,19,25]$. Qin et al. zeigten in einer Metaanalyse, dass diese Konzepte die Lehrumgebung der medizinischen Ausbildung verbessern können [17]. Witten-Herdecke berichtete als erste deutsche Universität über den Einsatz von problemorientiertem und einem damit fast vollständig studierendenzentrierten Lehrkonzept im medizinischen Bereich [9]. In der Literatur werden positive Erfahrungen und Chancen im Rahmen der Restrukturierung der Lehrveranstaltungen durch studierendenzentrierte Formate wie fallbasiertes Lernen oder POL beschrieben $[6,9,17]$. Ziel des POL ist es, die Lehrinhalte als Problem den Studierenden bewusst zu machen und für dieses eigenständig unter Zuhilfenahme von Literatur eine entsprechende Lösung $\mathrm{zu}$ finden. Dadurch ergeben sich neue Fragestellungen, welche dann tiefergehend bearbeitet werden können [9]. Eine alternative $\mathrm{zu}$ POL ist das fallbasierte Lernen. Durch die Moderation können Missverständnisse oder fehlerhafte Lösungsansätze frühzeitig vermieden werden. Dadurch kann die Lehrstruktur effizienter und zielgerichteter als im POL erfolgen [7, 20, 22]. Mithilfe des fallbasierten Lernens kann dadurch eine Balance zwischen dozenten- und studierendenzentrierter Lehre erreicht werden [7]. Diese kann, je nach Bedarf, dynamisch im Rahmen der Lehrveranstaltung verändert werden [7]. Dadurch kann fallbasiertes Lernen auch bei geringerem Vorwissen und Zeitlimitationen eingesetzt werden, während POL häu- 
Tab. 1 Zeigt die Ergebnisse des Evaluationsbogens der Universität M2016 vs. M2017. Es erfolgte ein ungepaarter zweiseitiger $t$-Test, bei $p<0,05$ folgte ergänzend ein einseitiger Test

\begin{tabular}{|c|c|c|c|}
\hline & $\begin{array}{l}\text { Mittelwert } \\
\mathbf{M}_{2016} \\
\mathbf{M}_{2017}\end{array}$ & $\begin{array}{l}\text { Standardabwei- } \\
\text { chung }\end{array}$ & $p$-Wert \\
\hline \multirow{2}{*}{$\begin{array}{l}\text { Wie bewerten Sie die Veranstaltung insge- } \\
\text { samt? }\end{array}$} & 2,56 & 1 & \multirow[t]{2}{*}{$0,011^{*}$} \\
\hline & 2,23 & 0,91 & \\
\hline \multirow{2}{*}{$\begin{array}{l}\text { Wie gut wurden die angegebenen Lernziele } \\
\text { definiert? }\end{array}$} & 2,67 & 1,04 & \multirow[t]{2}{*}{$0,003^{*}$} \\
\hline & 2,25 & 1,01 & \\
\hline \multirow[t]{2}{*}{ Prüfungsrelevanz der Veranstaltung } & 2,28 & 1,01 & \multirow[t]{2}{*}{$0,007^{*}$} \\
\hline & 1,93 & 0,83 & \\
\hline \multirow{2}{*}{$\begin{array}{l}\text { Wie schätzen Sie die fachliche Kompetenz } \\
\text { des Dozenten ein? }\end{array}$} & 2,03 & 0,9 & \multirow[t]{2}{*}{$<0,001^{*}$} \\
\hline & 1,6 & 0,67 & \\
\hline \multirow{2}{*}{$\begin{array}{l}\text { Wie bewerten Sie Motivation und Vorberei- } \\
\text { tung des Dozenten? }\end{array}$} & 2,32 & 0,98 & \multirow[t]{2}{*}{$0,007^{*}$} \\
\hline & 1,97 & 0,91 & \\
\hline \multirow{2}{*}{$\begin{array}{l}\text { Hat der Dozent die Inhalte angemessen } \\
\text { wiederholt? }\end{array}$} & 2,39 & 0,86 & \multirow[t]{2}{*}{$<0,001^{*}$} \\
\hline & 1,97 & 0,79 & \\
\hline \multirow{2}{*}{$\begin{array}{l}\text { Wie bewerten Sie die Möglichkeit, Fragen zu } \\
\text { stellen, und die Bereitschaft zur Diskussion? }\end{array}$} & 1,87 & 0,95 & \multirow[t]{2}{*}{$0,007^{*}$} \\
\hline & 1,55 & 0,7 & \\
\hline \multirow{2}{*}{$\begin{array}{l}\text { Wie empfanden Sie die entstandene Lern- } \\
\text { und Arbeitsatmosphäre? }\end{array}$} & 2,31 & 0,98 & \multirow[t]{2}{*}{$0,006^{*}$} \\
\hline & 1,94 & 1 & \\
\hline \multirow{2}{*}{$\begin{array}{l}\text { Konnten Sie dem Tempo des Dozenten gut } \\
\text { folgen? }\end{array}$} & 2,09 & 0,87 & \multirow[t]{2}{*}{$0,03^{*}$} \\
\hline & 1,86 & 0,75 & \\
\hline \multirow{2}{*}{$\begin{array}{l}\text { Inwieweit wurden Bezüge zu aktuellen } \\
\text { Themen hergestellt? }\end{array}$} & 2,24 & 0,96 & \multirow[t]{2}{*}{0,051} \\
\hline & 2,01 & 0,83 & \\
\hline \multirow{2}{*}{$\begin{array}{l}\text { Wie bewerten Sie die Qualität der Unter- } \\
\text { richtsmaterialien und -medien? }\end{array}$} & 2,46 & 0,99 & \multirow[t]{2}{*}{0,23} \\
\hline & 2,35 & 0,99 & \\
\hline \multirow[t]{2}{*}{ Wie gut wurde fächerübergreifend gelehrt? } & 2,5 & 0,96 & \multirow[t]{2}{*}{0,071} \\
\hline & 2,29 & 0,84 & \\
\hline
\end{tabular}

fig ein umfassendes Eigenstudium mit Literaturrecherche und intensiver Vorbereitung benötigt [6].

Da die Hauptvorlesung Chirurgie im selben Semester stattfand, war das Vorwissen der Studierenden in Abhängigkeit vom Vorlesungsplan heterogen. Um die Einflussnahme des Dozenten möglichst $\mathrm{zu}$ reduzierten, ist das Vorwissen allerdings bei der klassischen Durchführung von problem- und fallbasierten Lehrkonzepten maßgeblich [12]. Da keine Veränderung des Gesamtcurriculums der Universität möglich war, musste das neue Lehrkonzept in das bereits bestehende Curriculum eingegliedert werden. Um dem Wissensunterschied vor dem Hintergrund der curricularen Rahmenbedingungen an unserer Fakultät zu begegnen, wurden zur Vorbereitung auf die Lehrveranstaltung Empfehlungen wie die Nutzung von eLearning-Angeboten über eine eigene Plattform $[11,12]$ und weiter- führende Literatur gegeben. Im Seminar selbst wurden bedarfsgerecht Hilfestellungen gegeben und das standardisierte Lehrmaterial (Klassifikationshilfen, Therapieschritte etc.) entsprechend verwendet. Dadurch war stets ein positiver subjektiver Lernerfolg gewährleistet. Unsere Ergebnisse legen nahe, dass dadurch der Effekt nicht maßgeblich beeinflusst wurde, wie sich an der durchgeführten Subgruppenanalyse ohne Bewertungsunterschied in diesen Gruppen zeigt.

Der Berufswunsch nach dem Seminar zeigte, dass $40 \%$ der Studierenden sich noch nicht sicher waren und $36 \%$ der Studierenden eine Weiterbildung in einem chirurgischen Fach nach dem Studium anstreben. Im nationalen Vergleich entsprach das hier untersuchte Kollektiv anderen Umfragestudien von Studierenden [8].54\% der Studierenden konnten durch das Seminar für das Fach Orthopädie und Unfallchirurgie motiviert werden. $\mathrm{Ob}$ es sich hierbei um einen nachhaltigen Effekt handelt, kann anhand unserer Ergebnisse nicht gesichert werden. Allerdings ist eher von einem Einmaleffekt auszugehen, da nur $14 \%$ der Studierenden eine Weiterbildung zum Facharzt Orthopädie/Unfallchirurgie nach dem Seminar anstrebten.

Die im neuen Curriculum verwendeten Fallbeispiele wurden anhand der DGUV Unfallstatistik 2016 so ausgewählt, dass 4 häufige Verletzungsmuster der jeweiligen Körperregion pro Seminartermin behandelt werden konnten. Dies entsprach den Inhaltsvorgaben des ursprünglichen Seminars. Im Rahmen des Seminars waren 20 Dozenten am Unterricht beteiligt. Durch diese erfolgte in $54 \%$ der Einsatz der erstellten, papierbasierten Fallkarten, welche in Kleingruppen bearbeitet und anschließend mit der gesamten Seminargruppe weiter diskutiert wurden. Die elektronische Kasuistik wurde dagegen in $92 \%$ der Fälle verwendet und in der Gruppe erarbeitet. Hierbei zeigte sich in der Bewertung des Seminars kein Unterschied zwischen den Gruppen, welche die papierbasierten Fallkarten verwendet hatten, gegenüber den Studierenden, welche die Kasuistik mittels elektronischer Präsentation besprochen hatten. Also konnte bereits durch die digitale Darstellung der Kasuistik und das gemeinsame Erarbeiten die unfallchirurgische Lehre verbessert werden. Dies zeigte sich auch in der Lernatmosphäre und der Diskussionsbereitschaft im Seminar. Beim fallbasierten Lehrformat zeigte sich eine Verbesserung, was im Einklang zu vorangegangenen Studien steht $[5,18]$. Durch erhöhte Kommunikation im Seminar können dann auch Inhalte besser und gezielter wiederholt werden, wodurch auch Lernziele als besser definiert empfunden wurden. Diese Art der Kommunikation führte zu einer als gesteigert wahrgenommenen Motivation der Dozenten. Diese wurde rein subjektiv bewertet, allerdings kann durch Diskussionen im weitestgehend dozentenunabhängigen Lehrformat ein lebhaftes Seminar stattfinden. Dadurch kann auch die unterschiedliche Erfahrung der Dozierenden aus subjektiver 

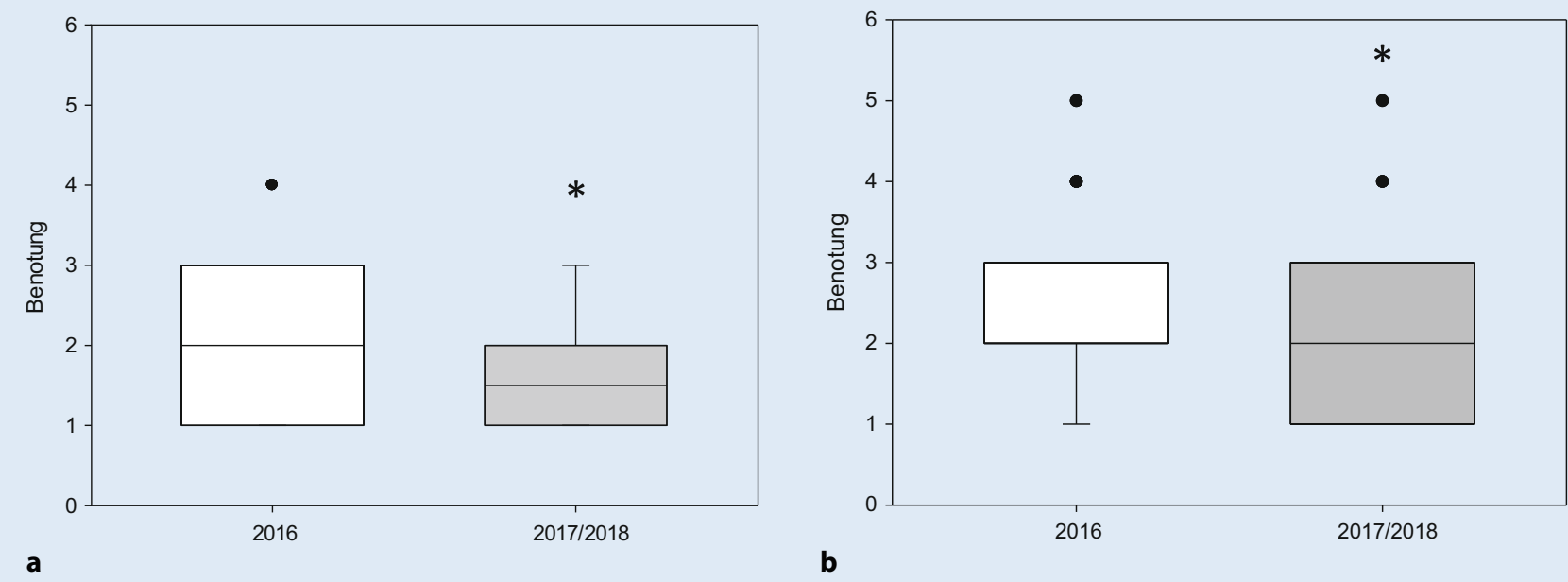

Abb. 2 A Boxplots zum Vergleich der Benotung zwischen den Jahren 2016 und 2017/2018 hinsichtlich eingeschätzter Kompetenz der Dozenten ( $\mathbf{a}$, Wie schätzen Sie die fachliche Kompetenz der Dozenten ein?) und Lern-/Arbeitsatmosphäre (b, Wie empfanden Sie die entstandene Lern- und Arbeitsatmosphäre?). Median, oberes, unteres Quartil, sowie Außenlieger ${ }^{*} p<0,05$

Sicht der Studierenden ausgeglichen werden.

Der überwiegende Teil der Studierenden empfand das fallbasierte Konzept als eine sinnvolle Lehrmethode. Allerdings waren $19 \%$ der Studierenden nicht von der Methode überzeugt. Dieser Effekt wurde auch in anderen, ähnlich konzipierten Studien beschrieben [18]. Dort zeigte sich keine Präferenz der Studierenden zu POL und somit studierendenzentrierter Methodik im Vergleich zur traditionellen Lehre [18]. In der Literatur wird die Ursache für die Ablehnung der Studierenden gegenüber studierendenzentrierten Lehrinhalten wie POL darin gesehen, das an den Universitäten meist dozentenzentriert gelehrt wird [13, 18]. Eine Umstrukturierung konnte in anderen Studien das positive Feedback für studierendenzentrierter Lehrmethoden deutlich erhöhen $[15,18,24]$. Eine weitere Möglichkeit, die Studierenden im Umgang mit eigenständigen, fallbasierten Lehrformaten zu fördern, aber auch Inhalte zu vertiefen, ist dabei, über die üblichen Seminare hinaus weitere Lehrinhalte und Kasuistiken über eLearning zur Verfügung zu stellen [11, 12].

Eine uneingeschränkte Wissensvermittlung durch fall- und problemorientiertes Lernen wird in der Literatur mitunter aber auch kritisch gesehen und ein reflektierter, gezielter Einsatz gefordert $[5,24]$. Auch an die Dozenten werden durch diese Lehrmethoden höhere Anforderungen gestellt [10]. Das starke Miteinbeziehen der Studierenden, v. a. hinsichtlich der Fallvorstellung und Diskussion, erfordert sinnvoll ergänzende Informationen, um das Auftreten von Missverständnissen oder gar die Verbreitung von Fehlern während des Seminars zu verhindern [9]. In dezidiert dafür angelegten Seminaren können Fälle, wie die hier vorgestellten, aber ein gutes Instrument sein, um Studierende in die Lage zu versetzen, Verletzungsmuster zu analysieren und Behandlungskonzepte festzulegen [24]. Diese Lernmethode bereitet die Studierenden auf das klinische Arbeiten vor [14]. In der Folge ist es vorstellbar, dass die fallbasierten Methoden zusätzlich mit Training an chirurgischen Simulatoren kombiniert wird, um auch den praktischen Aspekt der Ausbildung abzubilden.

\section{Limitationen}

Die Evaluationen dieser Studie erfolgte anhand der durch die Universität vorgegebenen Evaluationsbogen und anhand der spezifisch für die Neugestaltung erstellten Evaluation. Die spezifische Evaluation wurde nur in der Gruppe des neu etablierten Lehrkonzeptes durchgeführt, und nicht an der Kontrollgruppe aus dem Vorjahr. Die Dozenten setzten das neue Konzept um, allerdings waren die Fallkarten nicht in allen Seminaren verwendet worden, sodass dadurch Unterschiede in der Durchführung entstanden. Das elektronische Fallmaterial wurde dabei aber von über $90 \%$ der Dozierenden entsprechend eingesetzt. Hinsichtlich der Bewertungsqualität zwischen den papierbasierten und elektronischen Unterrichtsabläufen gab es in der Subgruppenanalyse allerdings keine Unterschiede, sodass davon auszugehen ist, dass ein rein elektronisches, fallbasiertes Kursformat ausreichend ist.

\section{Ausblick}

Die hier durchgeführte Untersuchung zeigte, dass durch fallbasiertes Lernen die Motivation der Studierenden erhöht werden kann. Durch das studierendenzentrierte Lehrkonzept können auch junge und somit weniger erfahrene Dozenten kompetenter wahrgenommen werden. Die Einführung solcher Lehrkonzepte ermöglicht es Studierenden, sich mit Erkrankungen und den Fällen intensiver zu beschäftigen. Diese Angebote können dann schrittweise z.B. zusätzlich in eLearning-Angebote eingebunden werden. Des Weiteren können die Fälle weiterentwickelt werden und später zu noch studentenzentrierterem problemorientiertem Lernen verwendet werden. Somit kann die Rolle des Dozenten schrittweise reduziert wer- 


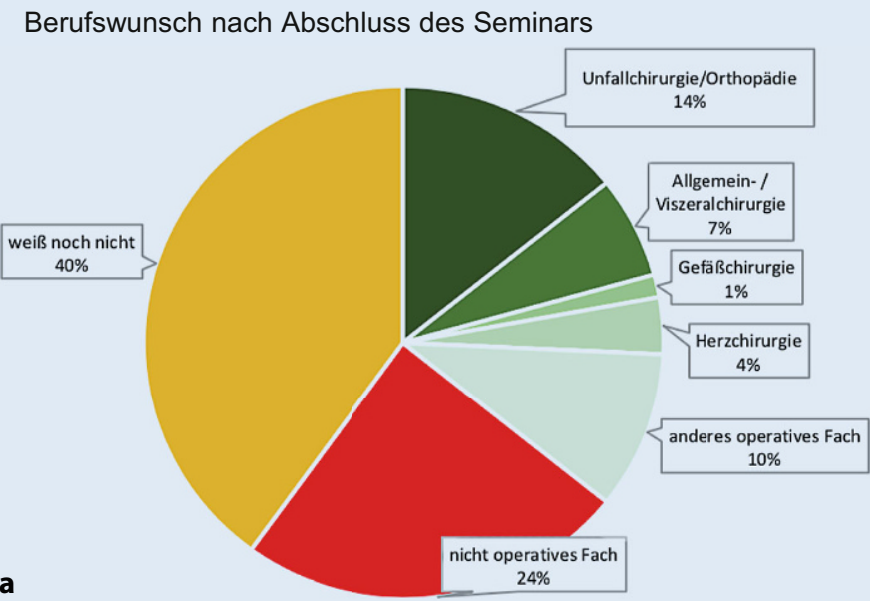

Hat Ihnen das fallbasierte Lernen in der Gruppe gefallen?

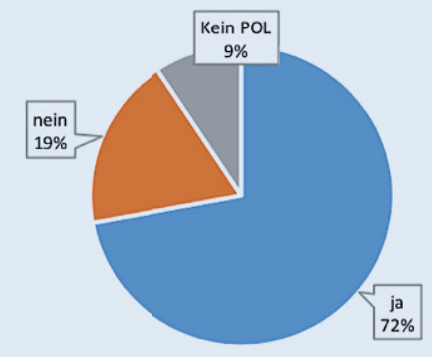

c

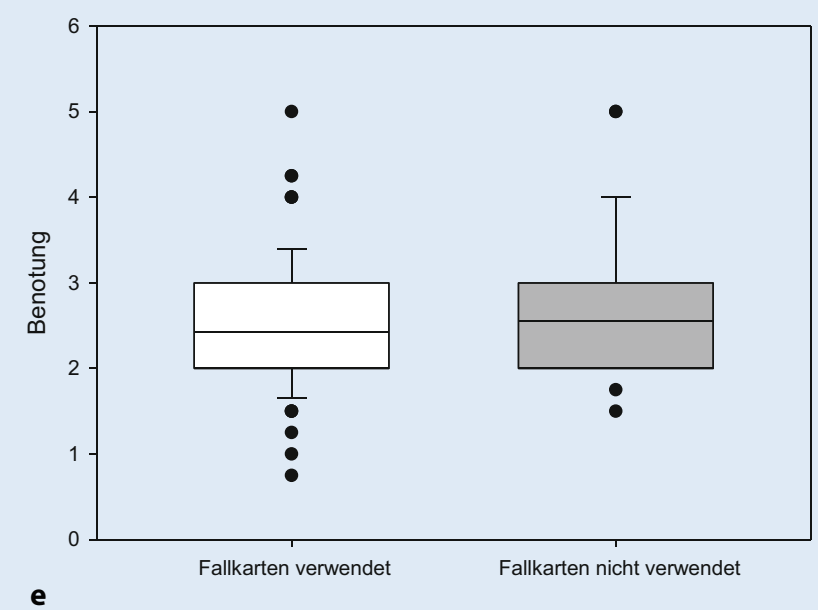

e
Fanden bzw. können Sie sich vorstellen, dass die vermittelten Lehrinhalte durch verschiedene Dozenten reproduzierbar sind?

b

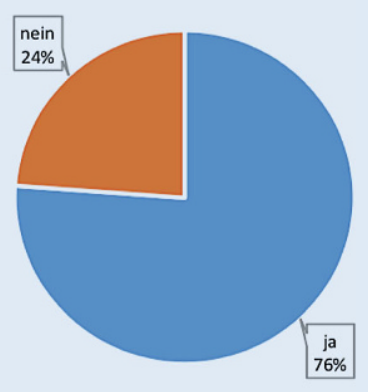

Der Unterricht wurde anhand der standardisierten Powerpoint-Fälle durchgeführt?

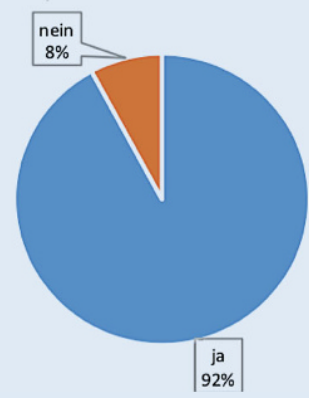

Abb. $3 \Delta$ Ergebnisse der Studierendenbefragung nach Abschluss des fallbasierten Seminars hinsichtlich Berufswunsch (a), Reproduzierbarkeit des Inhaltes (b), Gefallen an der Unterrichtsform (c) und Einsatz der elektronischen Fallpräsentation (d). Boxplots zum Vergleich des Benotungsunterschieds hinsichtlich des papierbasierten Fallkarteneinsatzes (e) sowie Vorlesungsbesuchs (f). Median, oberes, unteres Quartil, Außenlieger

den. Dies ermöglicht es, Studierende durch eigenständige Problemlösung auf die spätere klinische Tätigkeit vorzubereiten. Eine noch aus unserer Sicht unbeantwortete Frage ist, ob Seminarzeiten durch studierendenzentrierte Lehre effizienter genutzt werden können. Ein Großteil des vermittelten Lehrinhalts könnte dann bereits im Rahmen der Vorbereitung erarbeitet werden und dann im Seminar durch die Anwendung des Wissens vertieft werden.

\section{Fazit für die Praxis}

Durch das neu eingeführte fallbasierte Lehrkonzept im Praxisseminar Unfallchirurgie konnte eine studierendenzentrierte Lehre etabliert werden. Hierbei 
zeigte sich, dass das Konzept durch die Studierenden überwiegend positiv, insbesondere hinsichtlich der klinischen Relevanz und Lernatmosphäre, empfunden wurde und die wahrgenommene Motivation der Dozenten für das Seminar gesteigert werden konnte. Eine rein elektronische Fallvorstellung scheint dabei ausreichend. Standardisierte fallbasierte Unterrichtskonzepte in der Unfallchirurgie stellen in unseren Augen eine sinnvolle Ergänzung zu bestehenden Lehrmethoden dar.

\section{Korrespondenzadresse}

\section{Tobias Fritz, MD}

Klinik für Unfall-, Hand- und Wiederherstellungschirurgie, Universitätsklinikum des Saarlandes

Kirrberger Str. 1, 66421 Homburg/Saar, Deutschland

tobias.fritz@uks.eu

Funding. Open Access funding enabled and organized by Projekt DEAL.

\section{Einhaltung ethischer Richtlinien}

Interessenkonflikt. F. Strobel, T. Histing, T. Pohlemann, A. Pizanis, B.J. Braun, M. Orth und T. Fritz geben an, dass kein Interessenkonflikt besteht.

Für diesen Beitrag wurden von den Autoren keine Studien an Menschen oder Tieren durchgeführt. Für die aufgeführten Studien gelten die jeweils dort angegebenen ethischen Richtlinien.

Open Access. Dieser Artikel wird unter der Creative Commons Namensnennung 4.0 International Lizenz veröffentlicht, welche die Nutzung, Vervielfältigung, Bearbeitung, Verbreitung und Wiedergabe in jeglichem Medium und Format erlaubt, sofern Sie den/die ursprünglichen Autor(en) und die Quelle ordnungsgemäß nennen, einen Link zur Creative Commons Lizenz beifügen und angeben, ob Änderungen vorgenommen wurden.

Die in diesem Artikel enthaltenen Bilder und sonstiges Drittmaterial unterliegen ebenfalls der genannten Creative Commons Lizenz, sofern sich aus der Abbildungslegende nichts anderes ergibt. Sofern das betreffende Material nicht unter der genannten Creative Commons Lizenz steht und die betreffende Handlung nicht nach gesetzlichen Vorschriften erlaubt ist, ist für die oben aufgeführten Weiterverwendungen des Materials die Einwilligung des jeweiligen Rechteinhabers einzuholen.

Weitere Details zur Lizenz entnehmen Sie bitte der Lizenzinformation auf http://creativecommons.org/ licenses/by/4.0/deed.de.

\section{Literatur}

\section{Verwendete Literatur}

1. AntepohI W, Domeij E, Forsberg P (2003) A followup of medical graduates of a problem-based learning curriculum. Med Educ 37:155-162

2. Berdel WE, Zühlsdorf M, Nippert RP, Marschall B, Wilhelm MJ, Stümpel F et al (2001) Problemorientiertes Lernen-interdisziplinare Tumormedizin (poL-iT): ein integriertes Modell der Universitat Munster [Problem-based learning-interdisciplinary tumor medicine: an integrated model of the Munster University]. Onkologie 24(6):587-594. https://doi.org/10.1159/000055148

3. Björck U (2004) Distributed problem-based learning. Studies of a pedagogical model in practice. Göteborg studies in educational sciences 221. Acta Universitas Gothoburgensis, Göteborg

4. Donner RS, Bickley H (1993) Problem based learning in American medical education: an overview. Bull Med Libr Assoc 81:294-298

5. De Grave WS, Schmidt HG, Boshuizen HPA (2001) Effects of problem-based discussion on studying a subsequent text: a randomized trial among first year medical students. Instr Sci 29:33-44

6. Gwee MCE (2009) Problem-based learning: a strategic learning system design for the education of Healthcare professionals in the 21st century. Kao Hsiung I Hsueh Ko Hsueh Tsa Chih 25:231-239

7. Häusler J (2020) Unterrichtsqualität fallbasierter Seminare im Medizinstudium. Eine Videostudie. Dissertation. Technische Universität, München

8. Jacob R, Kopp J, Fellinger P (2018) Berufsmonitoring Medizinstudierende 2018 - Ergebnisse einer bundesweiten Befragung. Bericht der Universität Trier, Kassenärztliche Bundesvereinigung

9. Kahlke W, Kaie A, Kaiser H, Kratzert R, Schöne A, Kirchner V, Deppert K (2000) Reform des Medizinstudiums: Problemorientiertes Lernen - Eine Chance für die Fakultäten. Dtsch Arztebl 97(36):A2296/B-1959/C-1842

10. Kaufman DM, Holmes DB (1996) Tutoring in problem-based learning: perceptions of teachers and students. Med Educ 30:371-377

11. Knopp W, Ziegler R, Hohenberg G, Wendorf A, Redies M, Pohlemannt T (2008) MEC.O-Medical education online. Ein Schlüssel zur Wissenserweiterung in der unfallchirurgischen Studierendenausbildung im Rahmen der neuen Approbations ordnung für Ärzte. Vortrag: E-Learning-Day der Universität des Saarlandes, Saarbrücken

12. Knopp W, Ziegler R (2010) Inhalte der Lehr- und Lernplattform. Vortrag Deutscher Kongress für Orthopädie und Unfallchirurgie, Berlin

13. Lieberman SA, Stroup-Benham CA, Peel J, CampMG (1997) Medical student perception of the academic environment: a prospective comparison of traditional and problem-basedcurricula. Acad Med 72(10Suppl1):S13-S15

14. Mann KV (1994) Educating medical students lessons from research in continuing education. Acad Med 69:41-47

15. McParland M, Noble LM, Livingston G (2004) The effectiveness of problem-based learning compared to traditional teaching in undergraduate psychiatry. Med Educ 38(8):859-867

16. O'Neill PA, Willis SC, Jones A (2004) A model of how students link problem-based learning with clinical experience through „elaboration. Acad Med 77:552-561

17. Qin Y, Wang Y, Floden RE (2016) The effect of problem-based learning on improvement of the medical educational environment: a systematic review and meta-analysis. Med Princ Pract 25:525-532

18. Ratzmann A, Wiesmann U, Proff P, Kordaß B, Gedrange T (2013) Studentische Rezeption Zum Problemorientierten Lernen Im Vergleich Mit Konventionellen Lernmethoden Im Zahnmedizinischen Kieferorthopädischen Curriculum - Eine Pilotstudie. GMSZ Med Ausbild 30:1-17

19. Rüdiger M, Müller S, Neuwirth A, Kordonouri O, Guthmann F (2003) Integrating problem-based learning into traditional medical courses: the PBL approach to pediatrics in Berlin. Z Arztl Fortbild Qualitatssich 97:339-341

20. Setia S, Bobby Z, Ananthanarayanan P, Radhika M, Kavitha M, Prashanth T (2011) Case based learning versus problem based learning: a direct comparison from first year medical students perspective. Webmedcentral Med Educ 2:1-14

21. Spaulding WB (1969) The undergraduate medical curriculum (1969 model): mcmaster university. Can Med Assoc J 100:659-664

22. Srinivasan $M$, Wilkes $M$, Stevenson $F$, Nguyen $T$, Slavin S (2007) Comparing problem-based learning with case-based learning: effects of a major curricular shift at two institutions. Acad Med 82(1):74-82

23. Steinert Y (2004) Student perceptions of effective small group teaching. Med Educ 38:286-293

24. Thistlewaite JE, Davies D, Ekeocha S, Kidd JA, Macdougall C, Matthews P, Purkis J, Clay D (2012) The effectiveness of case-based learning in health professional education. ABEME systematic review: BEME guide No. 23. Med Tech 34(6):e421-e444

25. Yeo S, Chang BH (2017) Implementation of problem-based learning in medical education in Korea. Korean J Med Educ 29:271-282

26. DGUV Statistik (2017) https://publikationen.dguv. de/zahlen-fakten/schwerpunkt-themen/3382/ arbeitsunfallgeschehen-2016

\section{Weiterführende Literatur}

27. Kaufmann DM (1985) Implement problem-based learning in medical education: lessons from successful innovations. Springer, New York

28. St Pierre M, Breuer G (2013) Simulation in der Medizin, 2. Aufl., S116 